\title{
Saavedra, Villa Ortúzar, Palermo, Spoon River: las traducciones de Borges de Edgar Lee Masters en revista Sur
}

ZENÓN DEVIAGGE Universidad Nacional del Sur, Argentina / zendeviagge@gmail.com

\section{Resumen}

Este artículo examina tres poemas de Edgar Lee Masters que Jorge Luis Borges traduce en 1931 en la revista Sur. A partir del relevamiento de las condiciones específicas de un proyecto de traducción que desarrolla en su obra temprana, podemos considerar las traducciones de Masters como un posible punto de tensión entre el Borges «criollista» de la década del veinte y su posterior giro «universal».

Palabras clave: Sur / Jorge Luis Borges /

Edgar Lee Masters / Pedro Henríquez Ureña / estudios de traducción

\section{Saavedra, Villa Ortúzar, Palermo, Spoon River: Borges's translations of Edgar Lee Masters in Sur magazine}

Abstract

This article examines three poems of Edgar Lee Masters translated by Jorge Luis Borges in Sur magazine, in 1931. The research of specific conditions of a translation project developed in his early work allows us to consider those translations as a possible point of tension between a «criollista» Borges and his later «universal» turn.

Key words: Sur / Jorge Luis Borges / Edgar Lee Masters / Pedro Henríquez Ureña / translation studies

Recibido: 30/5/2019. Aceptado: 25/6/2019

Para citar este artículo: Deviagge, Z. (2019). Saavedra, Villa Ortúzar, Palermo, Spoon River: las traducciones de Borges de Edgar Lee Masters en revista Sur. El taco en la brea, 10 (junio-noviembre), 62-73. Santa Fe, Argentina: UNL. DOI: 10.14409/tb.v1i10.8686 
En sus inicios, Sur buscaba una dirección editorial definida. Desde 1931 a 1935 la periodicidad de las publicaciones era irregular, sin un formato uniforme (cfr. King:53-54). El proyecto inicial era el del americanismo,' principalmente en función de la iniciativa de Waldo Frank, Alfonso Reyes y Pedro Henríquez Ureña, quienes la pensaban como una plataforma de intercambio cultural entre América del Norte y América del Sur. La presencia de la literatura norteamericana en esa etapa es relevante, con numerosas traducciones de ensayos, relatos breves y poesía. En ese contexto, en el segundo y tercer número de la revista, en 1931, aparece por un período breve la sección «Tres poemas», en la cual Jorge Luis Borges traduce a los poetas modernistas Langston Hughes y Edgar Lee Masters. Las traducciones de Hughes han sido estudiadas, ${ }^{2}$ pero su trabajo con los textos de Masters «Ana Rutledge», «Petit, el poeta» $\mathrm{y}$ «Chandler Nicholas» apenas si ha recibido alguna mención ocasional por parte de la crítica, en una omisión cuanto menos llamativa. Es que esas traducciones, sostenidas a partir del programa esbozado en «Las dos maneras de traducir» $y$ apuntaladas por una serie de preocupaciones lingüísticas y formales recurrentes en los textos de Borges de la década del veinte, ofrecen uno de los mayores puntos de tensión entre el escritor «criollista» y el escritor «universal».

\section{Recepción y circulación de Edgar Lee Masters en América Latina de 1918 a 1931}

John Hallwas afirma que, en la poesía norteamericana, desde Leaves of Grass no hubo un volumen de poesía tan decisivo como Spoon River Anthology, de Edgar Lee Masters, cuya primera edición es de 1915 (cfr. Masters, 1992:1). Este libro, como se sabe, está compuesto por las voces de los más de doscientos cincuenta personajes enterrados en el cementerio de Spoon River, un pueblo ficcional del Middle West en el umbral de los siglos XIX y XX. Cada una de esas voces configura un poema que problematiza la categoría de «epitafio» que se le suele asignar. Como marca Jesús López Pacheco, primer traductor del libro completo al español, son más bien «autorretratos, autobiografías condensadas; a menudo son también alegatos, testimonios, confesiones, autodefensas y acusaciones póstumas» (Masters, 1993:14). Escritos en un verso libre despojado e irónico que recupera usos de la lengua cotidianos, los poemas de Spoon River conforman una obra conceptualmente innovadora que resume la historia de los Estados Unidos: «su expansión territorial, sus guerras, su destino manifiesto y la muerte lenta de sus valores preindustriales» (Masters 2010:4). El libro obtuvo un reconocimiento inmediato por parte de la crítica, ${ }^{3} \mathrm{y}$ muchos poemas de Spoon River fueron reseñados e incluidos en las antologías de poesía norteamericana de la época, como la Tendencies in Modern American Poetry, de Amy Lowell, publicada en 1917, o la de Louis Untermeyer, The New Era in American Poetry de 1921. Los poemas traducidos por Borges en Sur son tres. Por un lado «Anne Rutledge» $\mathrm{y}$ «Petit, the poet», los cuales pertenecen a la colección de Spoon River Anthology y fueron incluidos también en la antología de Lowell. ${ }^{4}$ "Chandler Nicholas», en cambio, es del volumen The New Spoon River, publicado en $1924 .{ }^{5}$ Spoon River Anthology fue traducido en su totalidad al alemán en 1924, en sueco en 1927, y en italiano en 1943, por Fernanda Pivano. ${ }^{6}$ La primera traducción completa al español estuvo a cargo del mencionado Jesús López Pacheco y de Fabio L. Lázaro, y fue publicada por Cátedra en 1993. Destacamos asimismo la versión de Alberto Girri, publicada en Buenos Aires por Editorial Fausto en 1979 y recientemente, en 2015, la de Rodrigo Olavarría, a cargo de la editorial chilena Das Kapital. ${ }^{7}$

Pero la primera traducción de Masters al español no es de Spoon River, un dato que muestra el estrecho vínculo que existía entre la difusión de la poesía norteamericana contemporánea con el panamericanismo como estrategia comercial de los Estados Unidos. Es el poeta nicaragüiense 
Salomón de la Selva, partícipe de la emergencia del modernismo norteamericano en la escena neoyorquina, quien traduce en 1918 «Silence» de Masters, poema que había sido publicado en Poetry apenas tres años antes. Lo hace con el título «El silencio» y forma parte de la sección «Pan American Poetry» de la revista The Pan American Magazine, la cual incluía artículos como «Peruvian Currency and Exchange» 0 «The Fruit Industry in Ecuador». ${ }^{8}$ La figura de Salomón de la Selva no le era ajena a Pedro Henríquez Ureña, quien en su artículo «Poetas de los Estados Unidos», publicado en Cuba en 1915, ya lo destacaba como «uno de los poetas de mayor promesa» de esa literatura (Roggiano:132). Clave en la recepción de la literatura norteamericana en Latinoamérica, la formación universitaria en Estados Unidos de Henríquez Ureña le brinda la posibilidad de estudiar de primera mano las innovaciones poéticas que allí se desarrollaban y de percibir tempranamente un «impulso de renovación completa» (128): «Con todo, nadie diría que los poetas norteamericanos de hoy se parecen a los de ayer. Los de ahora, en vez de la poesía refinada de 1900, piden inquietud, realidad, sangre...» (128). En Henríquez Ureña el panamericanismo toma una inflexión erudita que, a partir de una labor filológica y comparatista, identifica las novedades formales y temáticas del incipiente modernismo norteamericano en vistas de ampliar los repertorios de la poesía latinoamericana, tal como afirma Alfredo Grieco y Bavio:

El continuo interés que Henríquez Ureña puso siempre en esta literatura (y peculiarmente en su poesía) era filológico, pero en el sentido más amplio; parecía acreditar para la comparatística un improbable rasgo científico: la posibilidad de prever fenómenos. Conocedor de los efectos copernicanos que el parnasianismo y el simbolismo franceses habían tenido en Hispanoamérica sobre la lírica modernista, no le era imposible augurar las repercusiones incalculables de lo verbal que el conocimiento y expropiación de un nuevo orbe poético — por su apartamiento radical de una encendida tradición retórica de la que el modernismo en definitiva participaba — tendría sobre la poesía que se escribía en español. (684)

La labor crítica de Henríquez Ureña también sería fundamental para la publicación de la antología «La poesía norteamericana contemporánea» en la revista mexicana Prisma en el año 1922. Allí Salvador Novo también traduciría el poema «Silence» de Masters. Algunos años más tarde, Henríquez Ureña se instalaría en Buenos Aires para ejercer su labor docente, donde en 1927 escribe para la revista Nosotros "Veinte años de literatura norteamericana», texto que al año siguiente formaría parte de su libro Seis ensayos en busca de nuestra expresión y donde hay una breve reseña de Spoon River Anthology:

La nota fúnebre suena como punto de órgano en la obra famosa de Masters, la Spoon River Anthology: en cada poesía cuenta la vida de uno de los habitantes del pueblo de Spoon River, dormidos en el cementerio. Pero en Spoon River la vida no está decrépita como en los pueblos del norte de Boston: en medio de sus estrecheces, bulle de actividades y esperanzas. (82)

Los desplazamientos geográficos de Henríquez Ureña por el continente americano son también los de un intermediario transnacional a lo largo de un sector específico del sistema literario mundial. ${ }^{9}$ Del mismo modo, el crítico dominicano desempeñaría esa labor como miembro del comité de redacción extranjero de Sur, en el que se afianzaría, junto con Waldo Frank y Alfonso Reyes, como uno de los principales impulsores y difusores del panamericanismo y de la literatura 
norteamericana en la revista. ¿Es posible aventurar que es a partir de los desplazamientos cosmopolitas de Ureña y su posterior llegada a Buenos Aires que Borges conoce a Masters? El solo hecho de poder formular esta pregunta especulativa ${ }^{10}$ es suficiente para afirmar que la labor de divulgación de la poesía modernista norteamericana de Henríquez Ureña representa una de las claves fundamentales para la recepción de esa poesía en América Latina.

\section{Condiciones para un proyecto de traducción}

Existe sin embargo un dato que revela un primer contacto entre Borges y Masters anterior a las traducciones en la revista Sur. En una de las primeras reseñas de Fervor de Buenos Aires, publicada en España en 1924, el crítico Enrique Díez-Canedo, además de distinguir los versos de Borges «entre los de todos sus compañeros por la seguridad rítmica, por la riqueza verbal, por el desdén del nuevo lugar común» (Díez-Canedo:433) reconoce en Masters un precursor: «Y arrancada de los epitafios de la Spoon River Anthology parece esta «Inscripción sepulcral» en que se abrevia toda una vida, procedimiento favorito de Edgar Lee Masters» (433)." ¿Qué sucede en la obra de Borges entre el comentario de Díez-Canedo y las traducciones de Masters? ¿Cuáles son los desplazamientos que permiten vislumbrar el trayecto que lo lleva a esas traducciones? Esta última pregunta no es menor, en tanto no concebimos la traducción como un hecho aislado, anecdótico y lateral que ocasionalmente «dialoga» con el resto de la producción de Borges. Por el contrario, seguimos la noción propuesta por Antoine Berman, para quien las traducciones representan un tipo de textos con espesor y especificidad propios que resultan de una minuciosa labor crítica previa ${ }^{12}$ enmarcada, a su vez, dentro de los contornos de un proyecto:

Toda traducción consistente se lleva a cabo a partir de un proyecto (...) El proyecto define la manera a partir de la cual el traductor va a llevar a cabo la transferencia literaria, cómo se encargará de la traducción misma y de la elección de un «modo» y un «estilo» de traducción. (6o; la traducción es nuestra)

¿Hay en la obra inicial de Borges indicios de un proyecto que, de algún modo, organice sus traducciones de Edgar Lee Masters en 1931 ? $^{13}$ En principio, hay dos ejes que vertebran un programa posible. El primero es el vínculo entre la lengua «criollista» y el Buenos Aires «orillero». Borges reivindicará la búsqueda del «no escrito idioma argentino» (Narvaja de Arnoux y Bein:23) apelando al carácter variable de la lengua, cuyo decurso a lo largo del tiempo no es como el de un afluente cristalino y puro sino que se asemeja al de «largos ríos cuyo caudal es turbio y revuelto» (Borges 1993:161). Esa lengua, «edificadora de realidades» (48), es el material a partir del cual Borges crea una Buenos Aires situada en el límite ficcional de la pampa y el arrabal, territorios que funcionan menos como accidentes geográficos que como superficies poéticas: ${ }^{14}$

Aquí no se ha engendrado ninguna idea que se parezca a mi Buenos Aires, a este mi Buenos Aires innumerable que es cariño de árboles en Belgrano y dulzura larga en Almagro y desganada sorna orillera en Palermo y mucho cielo en Villa Ortúzar y proceridá taciturna en las Cinco Esquinas y querencia de ponientes en Villa Urquiza y redondel de pampa en Saavedra. (Borges 1993:13)

El otro eje que apuntala el proyecto de traducción de Borges es, justamente, su reflexión acerca de esa práctica. En «Las dos maneras de traducir», de 1926, Borges inaugura «una fluida concepción entre lo escrito y sus contextos» (Waisman:51), poniendo el foco en los desplazamientos espacio- 
temporales de los textos y de la lengua. Entre la traducción de prosa y verso, la premisa de la «equivalencia» presenta más dificultades en la poesía, en la cual prevalece el ambiente, la connotación y el ademán por sobre el sentido (cfr. Borges 1997:256). Las dos maneras de traducir a las que alude en el título son definidas como la «romántica» y la «clásica». La primera practica la «literalidad» y prioriza la fidelidad al texto «original» por su condición de obra única, motivada por la reverencia al autor. Por otra parte, a los traductores clásicos que ejercitan la "perífrasis» les interesa «la obra de arte y nunca el artista» (257) y conciben el texto original menos como una creación irrepetible que debe respetarse al pie de la letra que como un repertorio a su disposición. Borges se inclina por esta «manera» cuando traduce a Masters, articulando en una relación productiva los repertorios formales del texto «original» con las posibilidades de una lengua literaria «criollista». Esos son, a grandes rasgos, los lineamientos generales de su proyecto de traducción para «Ana Rutledge», «Petit, el poeta» $\mathrm{y}$ «Chandler Nicholas». Proponemos entonces un breve recorrido por esas traducciones, teniendo en cuenta que «todo aquello que un traductor pueda decir o escribir acerca de su proyecto se vuelve realidad solamente en la traducción» (Berman:61).

\section{Las traducciones}

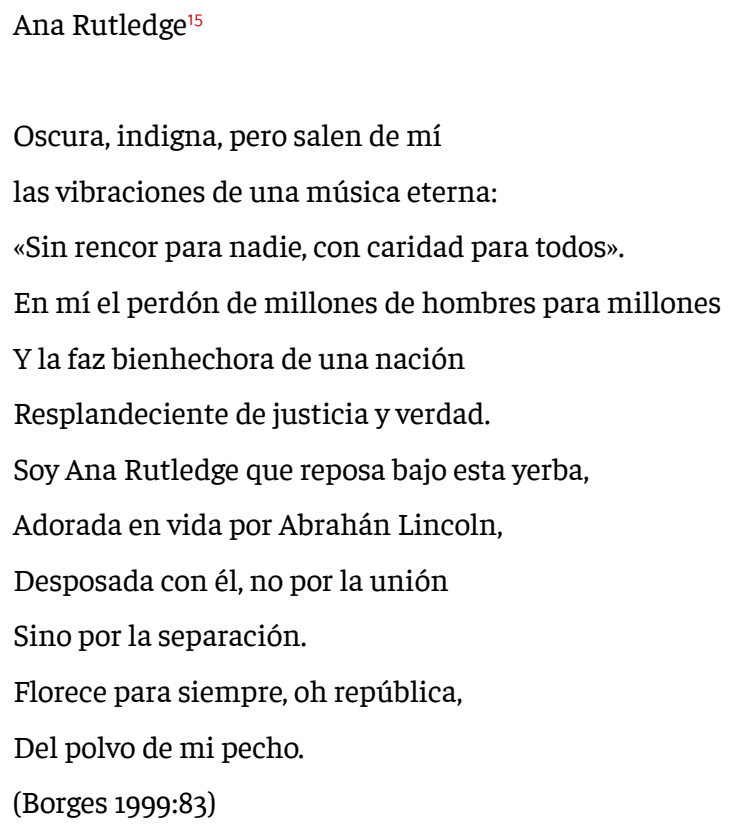

La figura de Anne Rutledge ostentaba durante la primera mitad del siglo XX un lugar privilegiado en la cultura popular norteamericana como el supuesto primer (y verdadero) amor de Lincoln, quien, se decía, nunca llegó a recomponerse completamente de su prematura muerte en 1835. No es para nada menor la traducción de Anne por su variante en español «Ana», y de Abraham por «Abrahán». Ya desde el título del primer poema Borges busca que los versos tengan «sabor a patria, como guitarra que sabe a soledades y a campo y a poniente detrás de un trebolar» (1925:19). Para lograr ese sabor, es necesario traducir incluso los nombres propios extranjeros: Jorge Federico Hegel, Jorge Bernardo Shaw, Eduardo Guillermo Lane o Ricardo Lamb, por ejemplo, aparecen en El tamaño de mi esperanza. Aunque la traducción de nombres propios era una práctica usual, en Borges es parte de una búsqueda deliberada de familiaridad con la lengua, para «conversar 
con los otros, con los muchachos querencieros y nuestros» (1993:11). Ocurre lo mismo en sutiles inflexiones lingüísticas, como la «yerba» que cubre la tumba de Ana Rutledge en el séptimo verso. El alto grado de territorialidad se condensa más por la musicalidad peculiar del yeísmo rioplatense que por el significado de la palabra, al igual que lo que sucede en «La promisión de alta mar», de Luna de enfrente, en el que el poeta evoca desde el océano «un atardecer de provincia, lacio como un yuyal» (2009:128; cursiva nuestra). Esas modulaciones en la traducción son también de orden semántico. La «yerba» se trama en una suerte de red botánica rioplatense que bosqueja las coordenadas textuales de la Buenos Aires borgesiana entre la pampa y el arrabal. ${ }^{16}$

En la biografía de Lincoln que publicó en 1931, Masters cuestiona la autenticidad del romance con Anne Rutledge, afirmando que en realidad la historia se basa en un rumor local expandido luego del ascenso de aquel al poder (cfr. Masters, 1931:46). El origen local de esa historia, los detalles que se agregan a medida que transita de boca en boca y la opacidad biográfica progresiva que adquiere la figura de Anne Rutledge (o Ana Rutledge) justifican la elección de Borges por el adjetivo "oscura» que da inicio al poema, privilegiando el ambiente y la connotación de las palabras por sobre la equivalencia estricta. ${ }^{17}$ "Oscura» repite asimismo la vocal del inglés out, tratando de corresponder la musicalidad del verso.

Con la inclusión del adversativo "pero» en el primer verso, ausente en el original en inglés, Borges hace de Ana Rutledge el motor invisible de la historia norteamericana. En la traducción ese adversativo funciona como el centro gravitatorio del poema, al intensificar y abismar la distancia entre la muchacha «oscura» e «indigna» que construyen los rumores del relato popular y la que se afirma como la fuente de la que emanan «las vibraciones de la música eterna», la depositaria del «perdón de millones de hombres para millones», la «faz bienhechora de una nación/ resplandeciente de justicia y verdad». Es el mismísimo polvo de su pecho, sus restos mortales consumidos en la tumba los que se convierten en la tierra fértil de la que florecen para siempre los valores republicanos. Esta idea de la «patria» como propiedad personal, familiar e íntima comprendida en un individuo es recurrente en varios textos de Borges. ${ }^{18}$ Con su traducción, la tumba de «Ana Rutledge» se deposita en un mausoleo de personajes laterales y familiares que desestabilizan y reordenan antiguos parnasos.

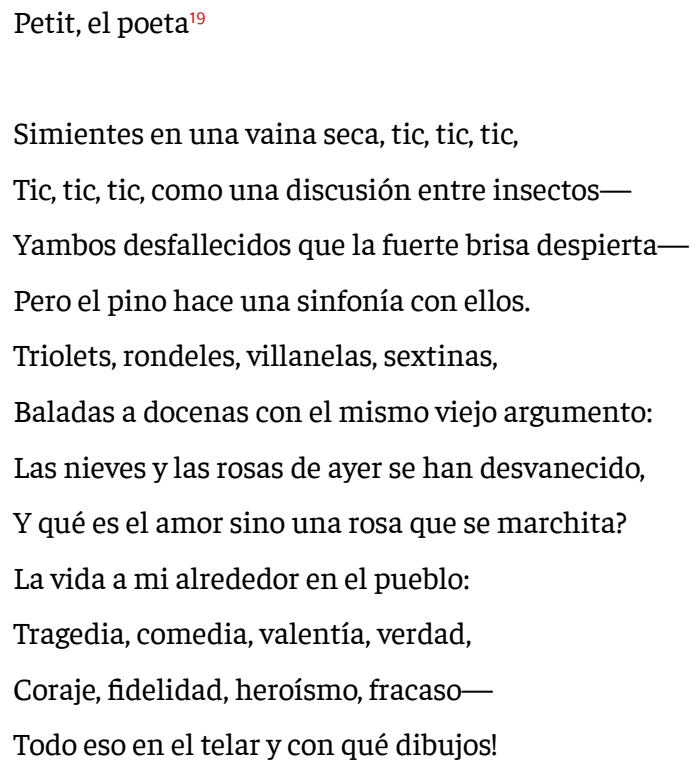


Monte, pastizales, ríos y arroyos-

Ciego toda mi vida a todo eso.

Triolets, sextinas, villanelas, rondeles,

Simientes en una vaina seca, tic, tic, tic,

Tic, tic, tic, qué minúsculos yambos,

Mientras Homero y Whitman rugían en los pinos!

(1999:83-85)

El poeta, desde un principio, no tiene ningún tipo de contemplaciones para con su propia obra, en la que los contenidos gastados hacen «tic» como semillas dentro de la «vaina seca» de la forma. En el quinto verso y al inicio del sexto, se enumera un pesado catálogo de variantes métricas fijas que, en el original de Masters, son un repliegue crítico hacia su obra temprana, durante la cual experimentó con esas formas poéticas. Aparece la voz francesa rondeau, que López Pacheco y Pivano adaptan respectivamente como rondó y rondò. Pero Borges la borra completamente y en su lugar coloca «sextina», en una alusión menos técnica que evocativa: no es solo un metro de la gauchesca, sino el que Martín Fierro utiliza para dar cuenta de sus recorridos por la pampa.

Para Petit, el «mismo viejo argumento», el del paso inexorable del tiempo, es en realidad la expresión resignada de un lugar común: «Las nieves y las rosas de ayer se han desvanecido/Y qué es el amor sino una rosa que se marchita?». Esas imágenes cargadas de fragilidad y evanescencia se neutralizan frente a una visión de la pampa, en una operación que se destaca aún más cuando se la compara con otras traducciones de ese fragmento. Tenemos en primer lugar la de López Pacheco (1993): bosques y prados, arroyos y ríos

La de Girri (1979), muy similar: bosques, prados, arroyos y ríos

La de Olavarría (2015):

bosques, planicies, riachuelos y ríos

Y finalmente, la de Borges:

monte, pastizales, ríos y arroyos

En las tres primeras traducciones «bosque» funciona más bien como un tópico atemporal. Pero la elección de palabra «monte» por parte de Borges tiene un matiz fuertemente territorial, propio de la lengua coloquial rioplatense, que designa la percepción de la vegetación silvestre dispersa y recortada sobre el horizonte de la pampa. Del mismo modo, así como el plural "pastizales» se vincula con la flora propia de la pampa, el uso de la palabra «arroyo» produce un efecto musical similar al de «yerba» en "Ana Rutledge». Esta aclimatación añade un nuevo nivel de coordenadas en el bosquejo de una geografía de «las orillas».

\author{
Chandler Nicholas ${ }^{20}$ \\ Bañándome cada mañana, afeitándome, \\ Vistiéndome después, \\ Pero nadie en la vida para alegrarse \\ Con mi trabajada apariencia. \\ Caminando cada día, respirando hondo
}


En pro de mi salud,

Pero la vitalidad ¿de qué me sirvió?

Adelantando cada día la mente

Con meditación y lectura,

Pero nadie con quien canjear sabidurías.

No era un ágora, no era un banco de liquidación

Para lo intelectual, Spoon River.

Buscando, pero no buscado de nadie:

Maduro, afable, utilizable, pero no utilizado.

Encarcelado aquí en Spoon River,

Menospreciado por los buitres mi hígado,

Devorándose solo.

(1999:85)

Graciela Montaldo identifica dos direcciones distintas en la recepción de la obra de Borges al principio de la década del treinta. Por un lado, como poeta, es el portavoz de su generación. Pero en su faceta de ensayista el carácter arbitrario y misceláneo de sus prosas genera cierto rechazo entre el público y los intelectuales porteños (cfr. Montaldo:217). La apuesta de Inquisiciones (1925), El tamaño de mi esperanza (1926) y El idioma de los argentinos (1928) de reconfigurar por completo la tradición literaria y experimentar con las posibilidades del criollismo no tiene el efecto esperado. Representa «una ruptura fuerte que - al no tener interlocutores - pasará a ser productiva sólo en las décadas posteriores» (219-220; cursiva nuestra). La hostilidad hacia Borges es manifiesta, como se lee en esta crítica de Ramón Doll: «Jorge Luis Borges, racial y temperalmente tan criollo, y en quien sospechamos que tantas esencias argentinas están guardadas, ha escogido una expresión, una prosa antiargentina, sin matices, ni acentos nacionales» (cit. por Siskind:76). La traducción de «Chandler Nicholas», el tercero y último de los «Tres poemas», se vuelve significativa a la luz de esta renuencia. La descripción de la rutina de un mejoramiento («bañándome cada día», «caminando cada día», «adelantando cada día la mente») afianza menos una autopercepción («maduro, afable, utilizable») que un rechazo («no utilizado, no buscado por nadie»). Esa es, acaso, la autofiguración de un Borges que publica sus libros, que escribe en las principales revistas literarias de Buenos Aires, que agita la polémica y no encuentra «nadie con quien canjear sabidurías». Pero «Chandler Nicholas» es algo más. Es un texto a mitad de camino entre el Borges que viene celebrando el suburbio porteño y el que en el número subsiguiente de la revista escribe el cáustico artículo «Nuestras imposibilidades». Si Spoon River, para Chandler Nicholas, «no era un ágora, un banco de liquidación/ para lo intelectual», tampoco Buenos Aires lo será para Borges en aquel texto: «Vuelvo, pues, a nuestro cotidiano argentino», escribe. «No inquiero su completa definición, sino la de sus rasgos más fáciles. El primero es la penuria imaginativa» (1999:118). En los últimos versos Nicholas se siente tan «encarcelado» en Spoon River como Borges en la capital. Este, visiblemente airado, escribe que las revistas de Buenos Aires son «tan desconocedoras de los cinco continentes y de los siete mares como solícitas de los veraneantes costosos a Mar del Plata» (119). La traducción de «Chandler Nicholas», intrascendente a primera vista, ${ }^{21}$ no pareciera sólo una feroz crítica al ambiente cultural porteño sino un posible cierre definitivo de la etapa criollista. El elogio a la capital y sus suburbios se topa con un límite y Borges avizora el horizonte de lo universal. 


\section{Notas}

1 Nora Pasternac ofrece un amplio panorama de la etapa americanista de Sur («El proyecto inicial: el americanismo» -5-88-). Entendemos el término «americanismo» tal como se utiliza en Sur como una inflexión intelectual y cultural con matices propios que deriva del «panamericanismo».

2 «Borges as translator» (Kutzinsky:100-111).

3 Entre esos comentarios destacamos el de Pound, «Webster Ford» (seudónimo de Masters al momento de la publicación de Spoon River Anthology) publicado en The Egoist en 1915: «At last! At last America has discovered a poet. (...) At last the American West has produced a poet strong enough to weather the climate, capable of dealing with life directly, without circumlocution, without resonant meaningless phrases. Ready to say what he has to say, and to shut up when he has said it. Able to treat Spoon River as Villon treated Paris in 1460".

4 En Introducción a la literatura norteamericana, publicado en 1967, Borges le dedica una breve entrada a Masters, en la que lo reconoce como uno de los principales innovadores de la poesía estadounidense del siglo XX. Escribe: «Integran este libro, que es una suerte de comedia humana, doscientos cincuenta epitafios o, mejor dicho, confesiones de otros tantos muertos de un obscuro pueblo de provincia, que nos revelan su intimidad. Ahí está Anne Rutledge, “adorada en vida por Abraham Lincoln, desposada con él no por la unión sino por la separación"; ahí está el poeta Petit, que, insensible a la vida que lo rodea, fabrica polvorientos triolets, "mientras Homero y Whitman rugían en los pinos"; ahí está Benjamín Pantier, a quien ha sostenido siempre el amor de su mujer, que no lo quería. La obra está escrita en verso libre y es la única importante que nos ha legado este autor» (1999:32). Los cuatro poemas que comenta en este fragmento Borges («Anne Rutledge», «Petit, the Poet», «Benjamin Pantier» y «Mrs. Benjamin Pantier», al cual alude tácitamente) están a su vez incluidos en la selección de diecisiete poemas que Lowell hizo de los doscientos cincuenta y cinco que componen Spoon River en su antología de 1917. La estadística favorece la hipótesis del libro de Lowell como una de las posibles fuentes de Borges para traducir a Masters.

5 Generalmente ignorado por la crítica y el público, y relegado en la historia literaria como la continuación fallida del texto capital de Masters, en la introducción a su reedición de 1968, el helenista Willis Barnstone hace una puesta en valor de esta segunda parte leyendo las relaciones intertextuales de los poemas con los epigramas de la Antología griega, utilizada ampliamente por Masters. Barnstone propone una relectura de The New Spoon River a partir de las preocupaciones de Masters de mostrar los efectos de la industrialización y la urbanización en el pueblo y sus habitantes. Dicha introducción es útil para revisar toda la obra poética de Masters (cfr. Masters, 1968).

6 La traducción de Pivano publicada por Einaudi en 1993 cuenta con un estudio crítico de la propia Pivano y otros tres de Cesare Pavese, quien le atribuye a Masters el título de "padre de la literatura moderna» (en Masters, 1993b:xxv).

7 Hay otras dos traducciones completas al español que con las que no contamos para este trabajo: la de Jaime Priede, editada en España por Bartleby en 2012, y la de Gerardo Gambolini, editada en Argentina por Ediciones En Danza en 2018.

8 Para una lectura más amplia del período de de la Selva en Estados Unidos, recomendamos el texto de Hulme, 2019. Asimismo, un trabajo reciente aborda, desde una óptica norteamericana, el intercambio cultural propiciado por el fenómeno del panamericanismo durante el siglo XX: Cándida Smith, 2017.

9 Pascale Casanova remarca la función fundamental que desempeñan esos «mediadores» en ese espacio: «Los grandes cosmopolitas (a menudo políglotas) son, en efecto, una especie de agentes de cambio, "cambistas" que se encargan de exportar de un espacio a otros textos cuyo valor literario, por ello, fijan» (Casanova:36)

$10 \mathrm{Si}$ bien las anécdotas no verifican la hipótesis, nos ayudan a imaginar un escenario posible: «Sonia Henríquez Ureña relata que su padre y Borges se divertían mucho conversando juntos, incluso con mi madre que disfrutaba del ingenio de Borges y ella misma era divertida y fantasiosa. En medio de esas charlas recitaban largas parrafadas de Góngora y Quevedo y muchos otros que por supuesto recordaban ambos de memoria...» (Henríquez Ureña de Hlito:135). En una carta a Alfonso Reyes, Henríquez Ureña narra una escena de vacaciones con Borges en 1930 que evoca la singularidad de esa cofradía: «El paisaje es el mejor de la provincia: desde San Pedro hasta la Vuelta de Obligado. Frente a la casa, el Paraná ha formado una gran isla... Entre la isla y la estancia hay un brazo de río, que en la tarde es morado tendiendo a malva. Allí remé y nadé ayer con Borges (buen nadador), y hombre capaz de soltarse y ser alegre y sencillo» (118). Al mismo tiempo, Borges expresa su estima hacia Henríquez Ureña de manera categórica: «Yo tengo la impresión 
—claro que es absurdo decir eso—-, pero tengo la impresión de que él había leído todo; de que sabía todo» (Borges, 2005:187; cursivas nuestras).

11 La importancia que tiene para Borges esta reseña se evidencia en su pedido expreso de reproducirla en la revista Nosotros ese mismo año, en Buenos Aires, y en el prólogo a la edición de Fervor de Buenos Aires de 1969, en donde recuerda que al libro, «por lo que prometía de algún modo, lo aprobaron generosamente Enrique Díez-Canedo y Alfonso Reyes».

12 En Toward a Translation Criticism: John Donne, Antoine Berman piensa el vínculo entre los textos literarios y la crítica como una correspondencia orgánica, en tanto las reverberaciones «infinitas» del texto se manifiestan y perpetúan en el tiempo a través de un acto crítico. Para Berman, el fruto de esa exigencia vital por parte de la obra literaria, citando una referencia de Schlegel utilizada por Benjamin, es una crítica que «completará la obra, la rejuvenecerá, la configurará nuevamente». La transferencia de una obra de un lenguaje a otro, es decir la traducción, constituye para Berman una de las múltiples formas que puede adoptar la crítica (cfr. Berman:27).

13 Las obras que Borges publica desde 1923 a 1931 son los poemarios Fervor de Buenos Aires (1923), Luna de enfrente (1925) y Cuaderno San Martín (1929), y los volúmenes ensayísticos Inquisiciones (1925), El tamaño de mi esperanza (1926) El idioma de los argentinos (1928) y Evaristo Carriego (1930). No nos parece redundante enumerar esas producciones porque entendemos que un proyecto de traducción es histórico, y, como se sabe, el hecho de que Borges haya reescrito, reelaborado o incluso suprimido fragmentos completos de esa obra inicial dificulta el análisis de las condiciones de posibilidad de ese proyecto de traducción. Por eso, no recurrimos a las obras completas «supervisadas», sino a la edición crítica al cuidado de Rolando Costa Picazo e Irma Zangaro, publicado por Emecé en 2009, la cual incluye, además de valiosas notas, las primeras ediciones de muchos de esos poemas posteriormente suprimidos. Para los poemas faltantes e inéditos, utilizamos Textos recobrados. 1919-1929, editado por Emecé en 1997.

14 En «Palabrerías para versos», la concepción del lenguaje como el «ordenamiento eficaz de [la] enigmática abundancia del mundo» (Borges 1993:46) tiene en el axioma «todo sustantivo es abreviatura» (47) no sólo una definición sino una tarea literaria, como escribe en los dos últimos versos de «Versos del catorce», de 1925: la de devolverle «a Dios unos centavos/ del caudal infinito que pone en mis manos» (Borges 2009:134). De ese caudal y esa abundancia, «de la riqueza infatigable del mundo» escribe Borges, «sólo nos pertenecen el arrabal y la pampa» (Borges, 1993:25).

15 Out of me unworthy and unknown/ The vibrations of deathless music;/ «With malice toward none, with charity for all»./ Out of me the forgiveness of millions toward millions,/ And the beneficent face of a nation/ Shining with justice and truth./ I am Anne Rutledge who sleep beneath these weeds,/ Beloved in life of Abraham Lincoln,/ Wedded to him, not through union,/ But through separation./ Bloom forever, $\mathrm{O}$ Republic,/ From the dust of my bosom!

16 Lo leemos en «La pampa y el arrabal son dioses» $y$ «Ascendencia del tango»:

«Yo mismo, incrédulo de mí, que en una casa del barrio de la Recoleta escribo estas dudas, fui hace unos días a Saavedra, allá por el cinco mil de Cabildo y vi las primeras chacritas y unos ombúes y otra vez redonda la tierra y me pareció grandísimo el campo» (Borges 1993:23; cursivas nuestras).

«El tango no es campero: es porteño. Su patria son las esquinas rosaditas de los suburbios, no el campo; su ambiente, el Bajo; su símbolo, el sauce llorón de las orillas, nunca el ombú» (Borges 1928:121; cursivas nuestras).

17 Por el contrario, en las traducciones al español de Casey (1947), Girri (1979), López Pacheco (1993) y Olavarría (2015) siempre se traduce unknown por «desconocida», del mismo modo que Pivano (1993) utiliza un adjetivo ignota con similar significado en italiano.

18 La labor "patriota» entramada con una escena familiar se repite en «Isidoro Acevedo», un poema que articula la faceta íntima de un abuelo de Borges jugador de truco con su participación en la guerra civil entre Buenos Aires y las provincias:

Adicto al diálogo ladino del truco,

alsinista y nacido del buen lado del Arroyo del Medio comisario de frutos del país en el mercado antiguo del Once, comisario de la tercera,

se batió cuando Buenos Aires lo quiso

en Cepeda, en Pavón y en la playa de los Corrales.

(...)

(Borges 2009:179)

19 Seeds in a dry pod, tick, tick, tick,/ Tick, tick, tick, like mites in a quarrel--/ Faint iambics that the full breeze wakens--/ But the pine tree makes a symphony thereof./ Triolets, villanelles, 
rondels, rondeaus,/ Ballades by the score with the same old thought:/ The snows and the roses of yesterday are vanished;/ And what is love but a rose that fades?/ Life all around me here in the village:/ Tragedy, comedy, valor and truth,/ Courage, constancy, heroism, failure--/ All in the loom, and oh what patterns!/ Woodlands, meadows, streams and rivers--/ Blind to all of it all my life long./ Triolets, villanelles, rondels, rondeaus,/ Seeds in a dry pod, tick, tick, tick,/ Tick, tick, tick, what little iambics,/ While Homer and Whitman roared in the pines!

20 Every morning bathing myself and shaving myself,/ And dressing myself./ But no one in my life to take delight/ In my fastidious appearance./ Every day walking, and deep breathing/ For the sake of my health./ But to what use vitality?/ Every day improving my mind/With meditation and reading,/ But no one with whom to exchange wisdoms./ No agora, no clearing house/ For ideas, Spoon River./ Seeking, but never sought;/ Ripe, companionable, useful, but useless./ Chained here in Spoon River,/ My liver scorned by the vultures,/ And self-devoured!

21 Este poema no forma parte de Spoon River Anthology, sino de su continuación, The New Spoon River, de 1924, al que Borges aludiría con ironía en una reseña de Masters la revista El Hogar, en 1936: «Edgar Lee Masters, desde entonces, [la publicación de Spoon River] ha publicado muchos libros de versos, con la esperanza de repetirlos. Ha imitado a Whitman, a Browning, a Byron, a Lowell, a Edgar Lee Masters. Del todo es vano: es, por antonomasia, el autor de la Antología de Spoon River» (Borges 1986:57).

\section{Referencias bibliográficas}

Berman, A. (2009). Toward a Translation Criticism: John Donne. Kent, Ohio: The Kent State University Press. (Obra original publicada en 1995). Traducción de F. Massardier-Kenney.

Borges, J. L. (1925). Inquisiciones. Buenos Aires: Editorial Proa.

- (1993) [1926]. El tamaño de mi esperanza. Buenos Aires: Seix Barral.

(1928). El idioma de los argentinos. Buenos Aires: Gleizer.

(1986). Textos cautivos. Ensayos y reseñas en «El Hogar» (1936-1939). Edición de Enrique Sacerio-

Garí y Emir Rodríguez Monegal. Buenos Aires: Tusquets.

- (1997). Textos Recobrados 1919-1929. Buenos Aires: Emecé.

(1999). Borges en Sur. Buenos Aires: Emecé.

(2005) En Diálogo I. Ciudad de México: Siglo XXI.

(2009). Obras Completas I (1923-1949). Edición Crítica. Anotada por Rolando Costa Picazo e Irma Zangara. Buenos Aires: Emecé.

Casey, A. (1947). Dos siglos de poesía norteamericana. Poetas blancos y negros de los EE. UU. Buenos Aires: Claridad.

Casanova, P. (1999). La República Mundial de las Letras. Barcelona: Anagrama.

Díez-Canedo, E. (1924). Fervor de Buenos Aires. Nosotros, 433-434.

Grieco y Bavio, A. (1998). Estudios angloamericanos de un romanista. Pedro Henríquez Ureña y la literatura norteamericana. En Abellán, J.L. y Barrenechea, A.M. (Coords.). Henríquez Ureña, Pedro. Ensayos: edición crítica. Madrid: ALLCA XX, Universidad de Costa Rica, 683-69o.

Henríquez Ureña, P. (1928). Seis ensayos en busca de nuestra expresión. Buenos Aires: Babel.

Henríquez Ureña de Hlito, S. (1993). Pedro Henríquez Ureña: Apuntes para una biografía. Ciudad de México: Siglo XXI.

Hulme, P. (2019). The Dinner at Gonfarone's. Salomón de la Selva and His Pan - American Project in Nueva York, 1915-1919. Liverpool: Liverpool University Press.

King, J. (1986). Sur. A study of the Argentine literary journal and its role in the development of a culture, 1931-1970. New York: Cambridge University Press. 
Kutzinski, V. (2012). The Worlds of Langston Hughes. Modernism and Translation in the Americas. Ithaca and London, Estados Unidos: Cornell University Press.

Masters, E.L. (1992) [1915]. Spoon River Anthology. An Annotated Edition. Edited and with an Introductions and Annotations by John E. Hallwas. Urbana and Chicago: University of Illinois Press.

(1968) [1924]. The New Spoon River. Introduction by Willis Barnstone. New York: The Macmillan Company.

(1931). Lincoln, The Man. Columbia: The Foundation for American Education.

(1979). Antología de Spoon River. Buenos Aires: Librerías Fausto. Selección, traducción y prólogo de Alberto Girri.

- (1993a). Antología de Spoon River. Madrid: Cátedra. Traducción de J. López Pacheco y F.L. Lázaro.

(1993b). Antologia di Spoon River. A cura di Fernanda Pivano. Con tre scriti di Cesare Pavese. Torino: Einaudi. Traducción publicada en 1943.

(2010). Antología de la Antología de Spoon River. Ciudad de México: Coordinación de Difusión Cultural, Dirección de Literatura, Universidad Nacional Autónoma de México. Selección, traducción y prólogo de Sandro Cohen.

(2015). Antología de Spoon River. Santiago de Chile: Das Kapital. Traducción de Rodrigo Olavarría.

Montaldo, G. (1989). Borges: una vanguardia criolla. En Montaldo, G. (Comp.) Yrigoyen, entre Borges y Arlt (1916-1930). Buenos Aires: Contrapunto, 215-230.

Narvaja de Arnoux, E. y Bein, R. (1997). «Posiciones de Borges acerca del idioma nacional». En Borges. Buenos Aires: Departamento de Extensión Cultural de la Biblioteca del Congreso de la Nación, 19-30.

Pasternac, N. (2002). Sur. Una revista en la tormenta. Los años de formación: 1931-1944. Buenos Aires: Paradiso.

Roggiano, A. (1961). Pedro Henríquez Ureña en los Estados Unidos. Ciudad de México: State University of Iowa Studies in Spanish Languages and Literature.

Siskind, M. (2007). El cosmopolitismo como problema político: Borges y el desafío de la modernidad. Variaciones Borges 24. Iowa: The University of Iowa.

Waisman, S. (2005). Borges y la traducción. Buenos Aires: Adriana Hidalgo. 